\title{
Segmentation Of Tumor Brain Based On The Colour
}

\author{
Awfa Hasan Dakheel \\ University of Babylon, Collage of Basic Education, Iraq \\ Awfa_d@yahoo.com
}

Recived : 2\10\2018

Revised : 7\10\2018

Accepted : $11 \backslash 10 \backslash 2018$

Available online : $\quad 15 / 11 / 2018$

DOI: $10.29304 / j q c m .2019 .11 .1 .450$

\begin{abstract}
:
The objective of this study is to present a method that aids in the diagnosis of diseases in brain due to tumors from MRI brain image whereby segmenting the brain tumor is done by using a novel algorithm that depends on the colour of the 2D image. Therefore, this work consists of three main stages, the first one is loading image into memory, and then the segmentation algorithm is applied. Finally, in order to obliterate the noise object, the 2D median algorithm is conducted. After applying the method the results which are obtained show better output to determine the tumor, simultaneously the diameter of the tumor can be calculated.
\end{abstract}

Keywords: Noise removal, Segmentation algorithm, 2D median algorithm, Tumor.

\section{Introduction}

One of the main causes of the increasing rate of mortality among children and adults is brain tumor, where a tumor is defined as any mass that is produced by abnormal growth may affect anyone irrespective of their age. It consequently might be alike for everyone. Tumors can destroy brain cells directly. The healthy cells can also be damaged indirectly by moving some brain parts, which results in "inflammation, brain swelling, and intracranial pressure" [1].
Brain tumors are classified into two types, the first is malignant type and the other is called benign. Malignant neoplasm is also called brain cancer, where malignant melorem hastily and often occupies or attracts the brain vigorous zones. By contrast, the benign brain tumors, which usually grow slowly, have no cancer cells at all [2]. 
The result of using computer technology is widespread and comprehensive in many life applications or areas such as medical decision support covering a wide range of medical fields, such as cancer research, heart disease, gastrointestinal tract, and brain disease. In the last century, computer-aided diagnosis (CAD) has gradually become an essential area of intelligent systems [3]. CAD becomes very important in many applications such as detection or classification of diseases.

In general, unusual deviations happening in organs and tissues can be detected early by means of a number of diagnostic and imaging techniques such as CT scans, MRI, X-rays, and ultrasound [4]. "Magnetic resonance imaging (MRI)" is considered to be one of the basic remedial methods frequently used to portray the construction and function of the human body in which it provides rich information on excellent soft tissue variation and is particularly useful in neuroscience[5].

The fragmentation of medical images is a major step and an introductory stage in the use of computer assistance. The success of medical image analysis depends largely on micro-image fragmentation algorithms. It has become clear that the exact division of the medical image is essential in the planning of radiotherapy, clinical diagnosis and treatment planning [6].

Image Segmentation is very important stage in images and interpretation, processing, image segmentation consists of extracting one or several objects of interest from a given image [7].

Broadly, the techniques of image segmentation can be categorized into Region Based method, Threshold method, Clustering method Region and Edge based method [8],[9].

The threshold method is one of the important and vastly used methods which recognizes foreground objects from the background for medical image segmentation. This can be achieved by the similarity of gray levels. To select an appropriate threshold value $\mathrm{T}$ in between two peaks Thresholded image $\mathrm{g}(\mathrm{x}, \mathrm{y})$ From the histogram of an image, can be defined as $g(x, y)=1$ if $f(x, y)>T(1)$

0 else

where $f(x, y)$ is the input image

So, the values associated with gray level are to be categorized as "black (0)" if they are less than $\mathrm{T}$, whereas the values exceeding $\mathrm{T}$ are to be the "white (1)". Threshold method is thereby is a means to obtain a binary image from gray level one being processed and altered [10].

The gold standard for performing segmentation is to manually delineate the object boundary [11]. Segmentation - as a means of medical capturing - plays a very paramount role in diagnosing apart from treating a lot of diseases. Still, [12] it is deemed a challenging job because of the slender dissimilarity along with "speckle noise" reflected in the photos.

To achieve this goal, several methods have been proposed for the use of image segmentation in the field of therapy, aiming to split the image into distinct areas so that determining the tumors will get possible [13] [14]. Some of authors depend on K-means clustering algorithm to extract the tumor such as [15]. The other uses the Threshold techniques [16], [17] or Histogram threshold technique that is achieved by the presumption that all pixels possess intensity rate below the predefined threshold's which belongs to a specific region.

This paper is structured as follows: section 1 to cover the introduction; section 2 to tackle the materials and methods; section 3 to discuss the results and finally, section 4 which is devoted to the conclusion.

\section{MATERIALS \& METHODS}

This section is devoted to presenting the proposed method that is in Figure1; it is divided into a set of steps and each is responsible for a specific job: 


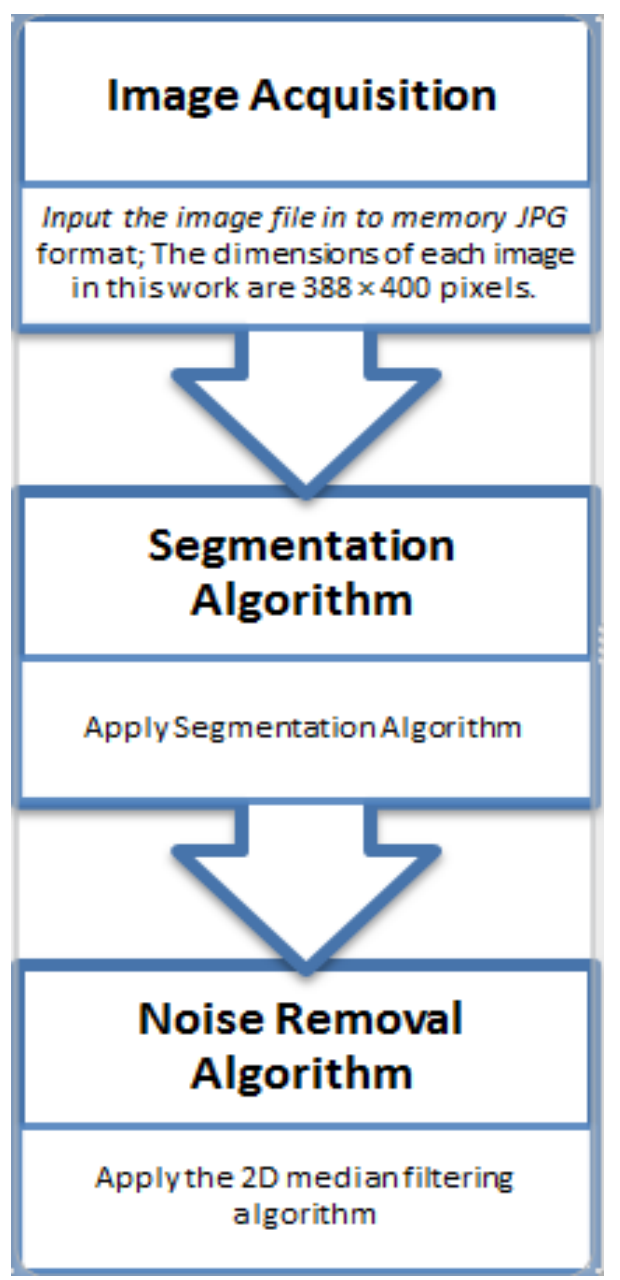

Figure 1: Main diagram of proposed method

\subsection{Image Acquisition}

At this procedure, the image file is uploaded to memory in $J P G$ format. A digital image is composed of a finite number of elements, each of which has a particular location and values. The elements of a digital image are called pixels. The dimensions of each image in this work are $388 \times 400$ pixels.

\subsection{Segmentation Algorithm}

Subsequently, the said algorithm will apply to have the tumor extracted from the brain. This study, in effect, depends on the tumor colour whose crucial role is represented by identifying the objects. The segmentation approach hence is elucidated by the algorithm below:

Firstly: Read the coloured photo.
Secondly: Spilt the prime colour matrix to 3 matrices [R], [G] and [B].

Thirdly: Calculate the mean and the standard deviation for each row.

Fourthly: Match a normal distribution object to the data.

Fifthly: Calculate the threshold $(95 \%$ confidence interval for the distribution parameters for each matrix Red, Green, and Blue).

Sixthly: Extract the (Red, Green, Blue) colours from the image by comparing each pixel for every matrix with threshold, if it is less than threshold, the pixel value is equal to zero, or else it is equal to one.

Seventhly: Apply noise removal algorithm.

\subsection{Noise Removal Algorithm}

Applying the "segmentation algorithm' is associated with the appearance of some objects which, besides being noisy, do vary in sizes and shapes. Subsequently, "2D median filtering algorithm" [18] is necessary to strip away those intrusive objects which must be tested by means of windows prior to the elimination process, as long as they are located within the window boundary. The algorithm is as mentioned in the study [19].

2D Median Filtering (Huang \& Yang, 1979) Algorithm:

allocate output Pixel Value[image width][image height]; allocate window[window width $\times$ window height];

edgex $=($ window width $/ 2)$ rounded down;

edgey $=$ (windowheight/ 2) rounded down;

for $\mathrm{x}$ from edge of image width - edge $x$

for $y$ from edgey to image height - edgey

$i=0$;

for $f x$ from 0 to window width

for $f y$ from 0 to window height

window $[i]:=$ inputPixe Walue $[x+f x-e d g e x][y+f y-e d g e y]$;

$i=i+1$;

sort entries in window!;

output pixel value $[x][y]=$ window[window width $\times$ window height $/ 2]$;

Eighthly: Return the coloured pixels to the whole matrix (Red, Green, Blue).

Ninthly: Compare the three matrices with 8 unit. 


\section{Result and discussion}

This part focuses on segmenting the tumor from the MRI brain image by using a novel algorithm that should run to extract the tumor . The proposed method implemented by using MATLAB application. In MATLAB application, in order to have the loaded MRI brain files of images read and stored in the memory, the user's border window should be opened immediately, as manifested in Figure 2:

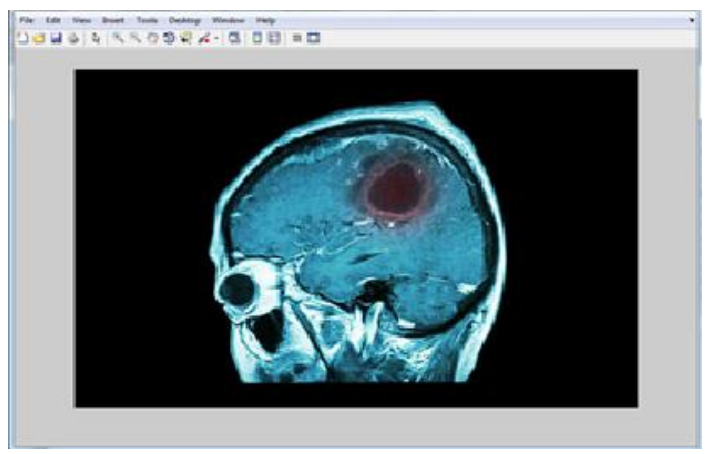

Figure 2: Represent The Original Image

This study depends on the colour of the tumor because it is a significant element to recognize the objects. Thus, after the images have been uploaded, the "segmentation algorithm" starts to extract the (Red, Green and Blue) colours from the image by comparing each pixel for every matrix with Threshold: if it is less than Threshold, the pixel value then is equal to zero or else it is equal to one. As shown in Figure 3 , that clarifies the result of the segmentation procedure:

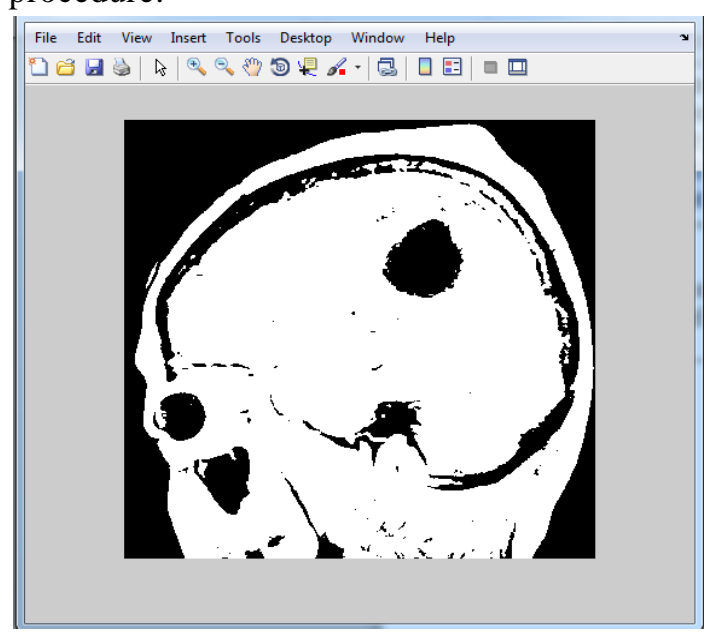

Figure 3: Apply segmentation algorithm.
In the subsequent process, the " $2 \mathrm{D}$ median filtering algorithm" is used to eradicate the noisy objects due to applying the segmentation algorithm .This is achieved through using windows to test the objects causing noise in the image. Hence, figure 4 shows the result:

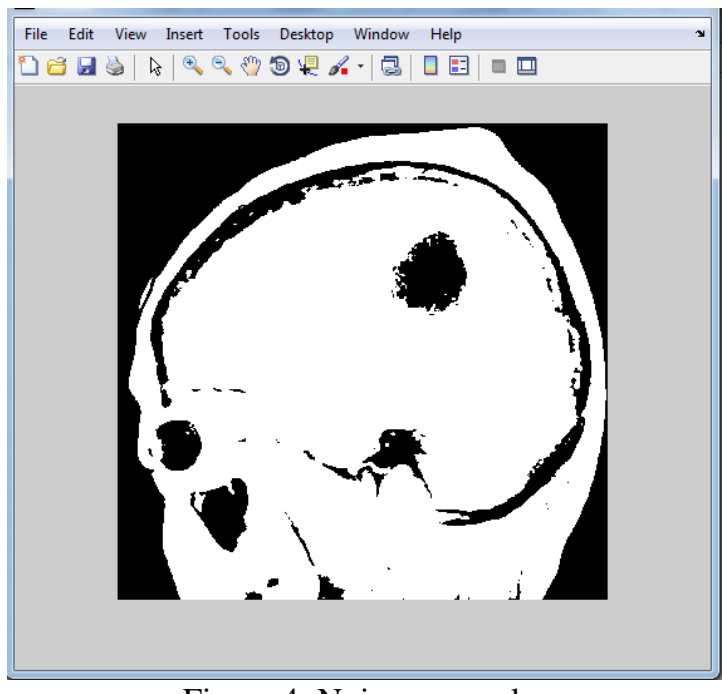

Figure 4: Noise removal.

In the end, a comparison should be made among the image pixels; all the pixels having (1) will maintain the original value of (Red, Green, and Blue) otherwise, they must have (0). Figure 5 displays that.

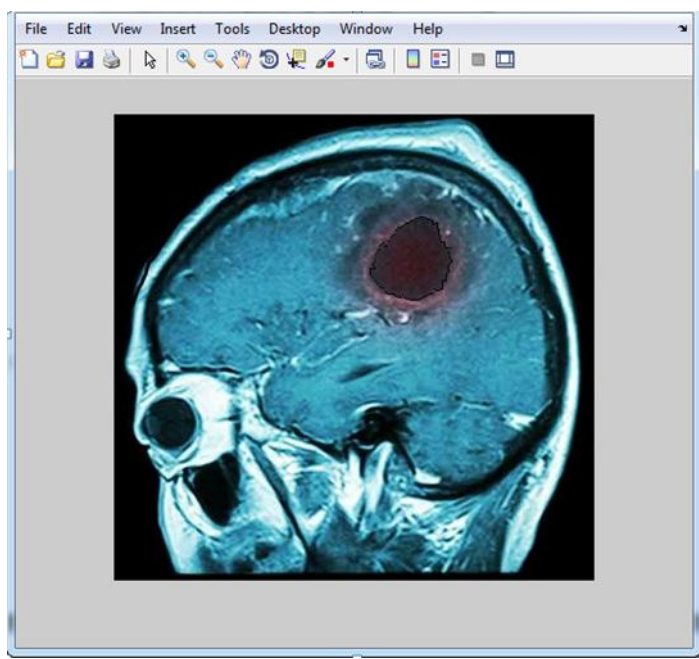

Figure 5: Tumor segmentation.

We were able to determine the tumor size, by calculating the diameter of the tumor .Therefore, a another results we can shown in Figure 6 : 


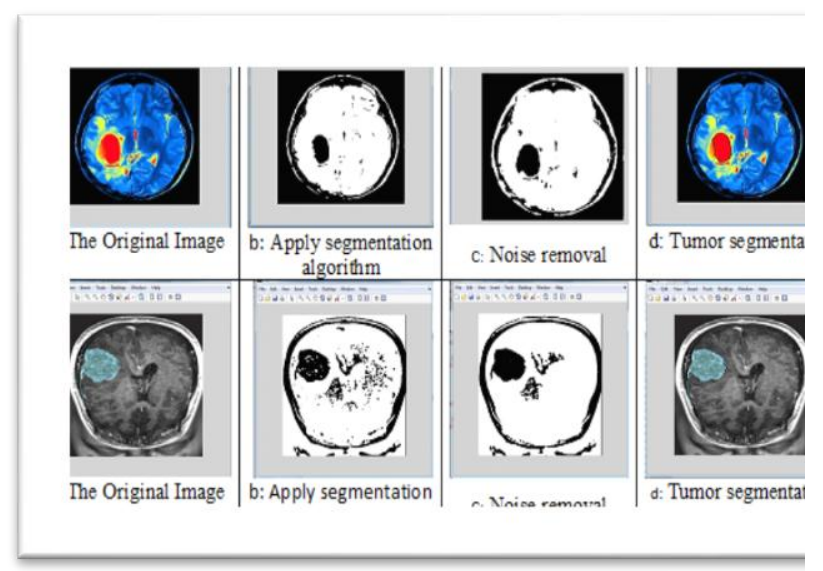

Figure 6: (a, b ,c ,d):Represent the result of the other images

As shown in Figure 6, the proposed method is applied in individual images to test it by using variant MRI brain images each one has got variant position and size of tumor in the brain, so in each case of MRI brain image, it is possible to determine and calculate the diameter of the tumor.

\section{Conclusion}

The proposed method is developed for segmenting the tumor which inflicts the brain from the brain MRI images. Therefore, the method that we have proposed is performed through multiple stages. The first stage is image acquisition, and with the second stage segmentation algorithm is to be applied; finally, we use the "2D median filtering algorithm" to get rid of the objects accompanied with noise. The proposed method applies on variant MRI brain images and each of these images contains a variant position and a specific size of the tumor. It is concluded that the proposed methods is effective and it can achieve promising results for determining the tumor and calculating its diameter.

\section{References}

[1] Ahmed Kharrat et al," Automated Classification of Magnetic Resonance Brain Images Using Wavelet Genetic Algorithm and Support Vector Machine", Proc. 9th IEEE Int. Conf. on Cognitive Informatics (ICCI'10), 2010.
[2] Bow S. et al, "Pattern Recognition and Image processing", Marcel Dekker, New York, USA, 2002.

[3] W.L.NADOS, R. D. KUMAR, "Medical Images Classification by using Artificial Intelligence Techniques, "International Journal of Engineering and Technology, Informatics, Vol.01,No. 01,2015, pp. 22-26.

[4]Noor E. A. ,et al ,"Comparative Study of Adaptive Network-Based Fuzzy Inference System, K-Nearset Neighbors And Fuzzy cmeans For Brain Abnormalities Segmentation ",Int. J. of Computers,Issue4,Vol.5, 2011.

[5] Shafaf I.,et al,"MRI Classification Abnormalities Segmentation Using K-Nearest Neighbors (K-NN)",Int. J. on computer science and engineering, Vol.3,No.2,2012

[6] El-Sayed A.EL.etal.m,"A Hybrid Technique For Automatic MRI Brain Images Classification ",Studia UNIV . Babes-Bolyai , Information ,Vol. 5,No.1, 2009.

[7] A.EL Allaoui .et al,"Evalution Algorithem For Segmentation Of Medical Images By Region Rrowing",13 th Internation Conference Computer Graphics and Visualization,2016.

[8] Prof. Dinesh D. Patil, "Medical Image Segmentation", international Journal of Computer science and Mobile Computer,Jan.2013,Vol.2,Issue1.

[9]J.Patel ,K.Doshi,"Study Of Segmentation Mothed for Detection of Tumor in brain MRI" ,ISSN2231-1297,2014,Vol.4,PP:279-284.

[10] Usha Rani .Nelakuditi ,T. Sri Rama Bharadwaja, N. Bhagiradh ,"Novel VLSI Architecture for Real Time Medical Image Segmentation ",IEEE Sponsored 2'nd International Conference On Electronics And Communication '2015),p.p:1048-1088.

[11] Houssem-Eddine Gueziri, Michael J. McGuffin, and Catherine Laporte,"Latency Management in Scribble-Based Interactive Segmentation of Medical Images",IEEE Transactions On Biomedical Engineering, VOL. 65, NO. 5, MAY 2018,pp. 1140-1150. 
[12]Neha. P. Raut , A.V.Gokhale," FPGA Implementation for Image Processing Algorithms Using Xilinx System Generator", IOSR Journal of VLSI and Signal Processing( IOSR-JVSP), Vol.2, Issue 4,May-June,2013, PP 26-36.

[13] P. H. S., S. H. K. B., and M. L. G, "Medical image segmentation," International Journal on Computer Science and Engineering, vol. 2, no. 4, pp. 1209-1218, 2010.

[14] N. W. L., K. R. D., N. M. A., and H. A. A, "Medical images classification by using artificial intelligence techniques," International journal of scientific engineering and technology research, no. 34, pp. 68126816, 2014.

[15] J.Katkar,T.Baraskar,V.R.Mankae"A Novel Approach for Medical Images segmentation using PCA and K-means Clustering", International Conference on Applied and theoretical Computing and Communication Technology(iCATccT) , 2015,pp. 430-435.
[16]Sahoo, Prasanna K., S. A. K. C. Soltani, and Andrew KC Wong. "Asurvey of thresholding techniques." Computer vision, graphics, andimage processing 41.2 (1988): 233-260.

[17] Wang, Rui, et al. "Threshold segmentation algorithm for automatic extraction of cerebral vessels from brain magnetic resonance angiography images.Journal of neuroscience methods 241 (2015):30-36.

[18] T. S. Huang and G. J. Yang, "A fast twodimensional median filtering algorithm," IEEE Transactions on Acoustics, Speech and Signal Processing, vol. 27, no. 1, pp. 13-18, 1979.

[19] N. A. Rasheed, W. L. Nados, "Object Segmentation from Background of 2D Image", Journal of University of Babylon, Pure and Applied Sciences ,Vol.(26), No.(5), 2018, pp. 204-215.

\section{تجزئة ورم الاماغ بناء على اللون \\ اوفى حسن دخيل \\ جامعة بابل ،كلية التربية الأساسية، العراق}

Awfa_d@yahoo.com

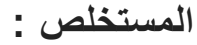

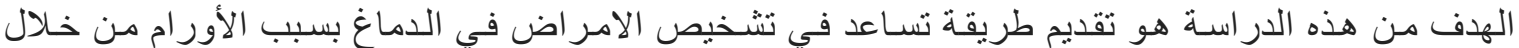

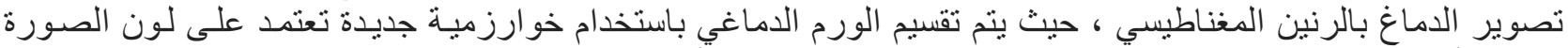

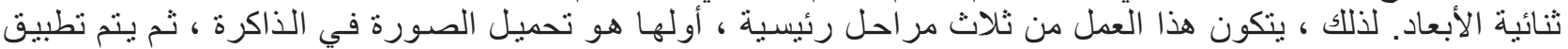

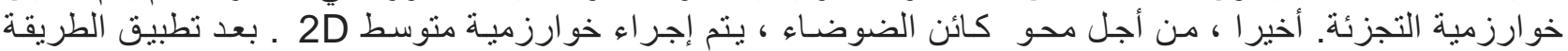

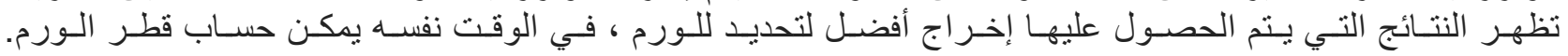$$
\text { كلمات البحث: إز الة الضوضاء ، خوارزمية التقسيم ، خوارزمية متوسط 2D ، ورم. }
$$ 